\title{
Prognostication of Cardiovascular Risk in Patients with Stable Coronary Heart Disease Depending on SYNTAX Score II on the Background of Nonalcoholic Fatty Liver Disease
}

\author{
Iryna Vakalyuk \\ Department of Internal Medicine of Stomatological Faculty, Ivano-Frankivsk National Medical University, Ukraine
}

Copyright $\mathrm{C} 2017$ by authors, all rights reserved. Authors agree that this article remains permanently open access under the terms of the Creative Commons Attribution License 4.0 International License

\begin{abstract}
Aim of the study was to evaluate the contribution of nonalcoholic fatty liver disease in predicting risk of major cardiovascular events by cumulative proportion surviving depending on the SYNTAX score II in patients with stable coronary heart disease. Materials and methods: Patients with stable coronary heart disease with and without nonalcoholic fatty liver disease underwent clinical examinations, electrocardiography, echocardiography, liver elastography, liver ultrasound, evaluation of the liver functional state. Then SYNTAX score I and II were calculated to all patients. Results: The two-year death-free survival ( $100.00 \%$ vs. $60.00 \% ; \mathrm{P}=0.0165)$, and a repeated nonfatal myocardial infarction-free survival (85.36\% vs. 33.33\%; P $=0.0045)$, and a stroke-free survival $(88.95 \%$ vs. $37.85 \%$; $\mathrm{P}=0.0021$ ), and a repeated revascularization-free survival ( $85.83 \%$ vs. $31.81 \% ; \mathrm{P}=0.0006)$ in patients with $\mathrm{SS}$ II $\geq 29$ and without nonalcoholic fatty liver disease were significant higher than in those with nonalcoholic steatohepatitis. Conclusions: the long-term prognosis of the patients with stable coronary heart disease is a most prognostically unfavorable on the background of SYNTAX score II $\geq 29$ and nonalcoholic steatohepatitis combination.
\end{abstract}

Keywords Stable Coronary Heart Disease, Nonalcoholic Fatty Liver Disease, Prognosis, SYNTAX Score

\section{Introduction}

Nonalcoholic fatty liver disease (NAFLD) was highly prevalent in patients with acute coronary syndrome [1]. It also has been associated with high cardiovascular risk and mortality in patients with coronary heart disease (CHD) [2-4]. The basis of nonalcoholic steatohepatitis (NASH), as one of the NAFLD stages, was the lipids accumulation, which caused hepatocytes cell damage, liver inflammation and fibrosis, and was correlated with atherosclerotic vascular [5]. Furthermore, the patients with hyperlipidemia were at the high risk of NAFLD, hence the need for their screening for the presence of steatosis or liver fibrosis [6].

In turn, SYNTAX score (SS) has been proposed as a predictor of clinical outcome following percutaneous coronary interventions in European and American revascularization guidelines [7-9]. Therefore, several clinical parameters [age, gender, creatinine clearance, left ventricular ejection fraction, presence of unprotected left main, peripheral vascular disease and chronic obstructive pulmonary disease] have been added to SS to obtain SS II $[10,11]$. Currently, SS II is able to predict a statistically significant difference in long-term outcomes between patients undergoing coronary artery bypass graft and those undergoing percutaneous coronary interventions $[12,13]$.

Several studies demonstrated correlation between NAFLD and SS [1]. However, SS II does not consider liver state. Therefore, the aim of the current study was to evaluate the contribution of NAFLD in predicting risk of major cardiovascular events by cumulative proportion surviving depending on the SS II in patients with stable CHD.

\section{Materials and Methods}

\subsection{Objectives}

Object of the study were 147 patients with stable CHD who have had an acute coronary syndrome with an indication of percutaneous coronary interventions more 
than 3 months ago. Participants were categorized into 3 groups according to the presence and severity of NAFLD: Group I - 88 patients without NAFLD; Group II - 36 patients with nonalcoholic steatosis; Group III - 23 patients with NASH. In each group individuals with SS II $<29$ and $\geq 29$, calculated at the time of acute coronary event, were identified. Stable CHD was defined according to the Unified Clinical Protocol "Stable ischemic heart disease"[14].

NAFLD was defined according to the EASL-EASDEASO Clinical Practice Guidelines for the management of NAFLD (2016), Unified Clinical Protocol "NASH" [15] and Adapted Clinical Guidelines "NAFLD" [16]. Patients who had the following conditions were excluded from the study: autoimmune, drug, alcoholic hepatitis and associated with $\mathrm{HBV}-, \mathrm{HCV}-, \mathrm{HDV}$-infection, an acute coronary syndrome less than 3 months ago, hemodynamically significant valvular lesions, chronic obstructive pulmonary disease, myocarditis, acute heart failure, other decompensated somatic states, cancer and lymphoproliferative diseases. The study was carried out according to the Helsinki declaration principles, and a written informed consent to coronary intervention and a two-year follow-up was obtained from all patients.

\subsection{Study Procedures}

General clinical examination, electrocardiography, echocardiography, liver elastography, liver ultrasound, evaluation of the liver functional state were performed to all patients. Each coronary lesion based on angiogram at the time of acute coronary event was scored to SS I, which was calculated with the SS online calculator [17]. To calculate SS II, the anatomical SS was combined with the following variables: age, gender, creatinine clearance, left ventricle ejection fraction, presence of peripheral vascular disease, unprotected left main and chronic obstructive pulmonary disease [15]. All patients were followed-up with telephone interviews and clinical investigation. Details about clinical outcomes were confirmed by reviewing hospital records or by the referring physician. Information about death, a repeated nonfatal myocardial infarction (MI), stroke and a repeated revascularization were prospectively collected.

\subsection{Statistical Analysis}

Survival analysis based on Kaplan-Meier curves was evaluated by a two-year cumulative proportion surviving $(\%)$, the difference between groups was determined by Cox's F and Gehan's Wilcoxon tests. In all cases, $\mathrm{P}$ values $<0.05$ were considered statistically significant. Statistical analysis was performed using Software «Microsoft Excel» and «Statistica» v. 10.0 StatSoft (USA).

\section{Results}

The 105 major cardiovascular events were experienced during a two-year clinical follow-up period (Table 1). Furthermore, the frequency of major cardiovascular events was dependent, not only, on the SS II value, but also the presence and severity of nonalcoholic fatty liver disease. In particular, a total of six deaths were observed. All of them were cardiac deaths and most of them were in patients with SS II $\geq 29$. If a one death occurred in Group II in patient with SS II $\geq 29$, then in Group III the five deaths were observed: two of them in patients' group of SS II $<29$ and three of them in patients' group of SS II $\geq 29$.

A repeated nonfatal MI occurred in 44 cases. In particular, a repeated nonfatal MI in patients' group of SS II $<29$ was in $6.45 \%$ patients of Group I vs. $35.00 \%$ patients of Group II vs.73.33\% patients of Group III $(\mathrm{P}<0.05)$. In patients' group of $\mathrm{SS}$ II $\geq 29$ a repeated nonfatal MI was more often and observed in $26.92 \%$ patients of Group I vs. $56.25 \%$ patients of Group II vs. $75.00 \%$ patients of Group III $(\mathrm{P}<0.05)$.

A total of 34 strokes were occurred during a study period. In patients' group of SS II $<29$ a stroke was observed more often in patients with NASH compared with patients with nonalcoholic steatosis $(60.00 \%$ vs. $30.00 \%$, respectively; $\mathrm{P}<0.05)$ or patients without NAFLD $(60.00 \%$ vs. $3.22 \%$, respectively; $\mathrm{P}<0.05$ ). In patients' group of $\mathrm{SS}$ II $\geq 29$ a stroke was observed in $62.50 \%$ patients of Group III, that was higher on $18.75 \%$ of Group II and on $43.27 \%$ of Group I respectively $(\mathrm{P}<0.05)$.

Table 1. A two-year clinical outcome of the study population according to the SYNTAX score II and NAFLD severity

\begin{tabular}{|c|c|c|c|c|c|c|}
\hline \multirow{2}{*}{$\begin{array}{c}\text { A two-year } \\
\text { follow-up outcome }\end{array}$} & \multicolumn{2}{|c|}{ No NAFLD $(\mathrm{n}=88)$} & \multicolumn{2}{c|}{ Nonalcoholic steatosis $(\mathrm{n}=36)$} & \multicolumn{2}{c|}{ NASH $(\mathrm{n}=23)$} \\
\cline { 2 - 7 } & SS II $<29(\mathrm{n}=62)$ & SS II $\geq 29(\mathrm{n}=26)$ & SS II $<29(\mathrm{n}=20)$ & SS II $\geq 29(\mathrm{n}=16)$ & SS II $<29(\mathrm{n}=15)$ & $\begin{array}{c}\text { SS II } \geq 29 \\
(\mathrm{n}=8)\end{array}$ \\
\hline Death & 0 & 0 & 0 & $1(6.25)$ & $2(13.33)$ & $3(37.50)$ \\
\hline $\begin{array}{c}\text { A repeated nonfatal } \\
\text { MI }\end{array}$ & $4(6.45)$ & $7(26.92)$ & $7(35.00)$ & $9(56.25)$ & $11(73.33)$ & $6(75.00)$ \\
\hline Stroke & $2(3.22)$ & $5(19.23)$ & $6(30.00)$ & $7(43.75)$ & $9(60.00)$ & $5(62.50)$ \\
\hline $\begin{array}{c}\text { A repeated } \\
\text { revascularization }\end{array}$ & $1(1.61)$ & $2(7.69)$ & $3(15.00)$ & $5(31.25)$ & $4(26.67)$ & $6(75.00)$ \\
\hline $\begin{array}{l}\text { Values are absolute and percentages in parentheses. NAFLD, nonalcoholic fatty liver disease; NASH, nonalcoholic steatohepatitis; MI, myocardial } \\
\text { infarction; SS II, SYNTAX score II }\end{array}$
\end{tabular}


A repeated revascularization was performed in 21 patients. In patients' group of SS II $<29$ a repeated revascularization was performed in $1.61 \%$ patients of Group I vs. $15.00 \%$ patients of Group II vs. $26.67 \%$ patient of Group III respectively $(\mathrm{P}<0.05)$. In patients' group of SS II $\geq 29$ a repeated revascularization was performed more often and was observed in $7.69 \%$ patients of Group I vs. $31.25 \%$ patients of Group II vs. $75.00 \%$ patient of Group III respectively $(\mathrm{P}<0.05)$.

In total, patients with NASH combined with SS II $\geq 29$ showed more worse cardiovascular outcome compared with those who had SS II $<29$ and more favorable duration of NAFLD.

The two-year major cardiovascular events-free survival was significantly lower in patients' group of SS II $\geq 29$ than in those with SS II <29.Moreover, the cumulative proportion was depended on the NAFLD course and was the lowest in patients with NASH. In particular, the two-year death-free survival $(100.00 \%$ vs. $78.57 \%$; $\mathrm{P}=$
0.0352) (Figure 1), and a repeated nonfatal myocardial infarction-free survival $(93.54 \%$ vs. $54.54 \%$; $\mathrm{P}=0.0176)$ (Figure 2$)$, and a stroke-free survival $(96.78 \%$ vs. $58.63 \%$; $\mathrm{P}=0.0058) \quad($ Figure 3$)$, and a repeated revascularization-free survival $(98.38 \%$ vs. $71.81 \%$; $\mathrm{P}=$ 0.0286) (Figure 4) in patients with SS II $<29$ of Group I were significant higher as compared with those of Group III (Table 2).

Similarly, a significant difference was found regarding the two-year major cardiovascular events-free survival of patients' group of SS II $\geq 29$ (Table 3). The two-year death-free survival $(100.00 \%$ vs. $60.00 \% ; \mathrm{P}=0.0165)$ (Figure 5), and a repeated nonfatal myocardial infarction-free survival $(85.36 \%$ vs. $33.33 \% ; \mathrm{P}=0.0045)$ (Figure 6), and a stroke-free survival $(88.95 \%$ vs. $37.85 \%$; $\mathrm{P}=0.0021) \quad$ (Figure 7), and a repeated revascularization-free survival $(85.83 \%$ vs. $31.81 \%$; $\mathrm{P}=$ 0.0006 ) (Figure 8) in patients with SS II $\geq 29$ of Group I were significant higher than in those of Group III.

Table 2. A two-year survival analysis according to the NAFLD severity in patients with stable CHD with SYNTAX score II $<29$

\begin{tabular}{|c|c|c|c|}
\hline \multirow{2}{*}{ A two-year follow-up outcome } & \multirow{2}{*}{ Cumulative proportion, $\%$} & \multicolumn{2}{|c|}{$P$ value } \\
\hline & & Gehan's Wilcoxon test & Cox's F test \\
\hline \multicolumn{4}{|c|}{ Death } \\
\hline No NAFLD (n=62) & 100.00 & \multirow{3}{*}{$\begin{array}{l}\mathrm{p}_{\mathrm{I}-\mathrm{II}}=0.6575 \\
\mathrm{p}_{\mathrm{I}-\mathrm{III}}=0.0352\end{array}$} & \multirow{3}{*}{$\begin{array}{l}\mathrm{p}_{\mathrm{I}-\mathrm{II}}=0.4568 \\
\mathrm{p}_{\mathrm{I}-\mathrm{III}}=0.0171\end{array}$} \\
\hline Nonalcoholic steatosis $(n=20)$ & 100.00 & & \\
\hline NASH $(n=15)$ & 78.57 & & \\
\hline \multicolumn{4}{|c|}{ A repeated nonfatal myocardial infarction } \\
\hline No NAFLD $(n=62)$ & 93.54 & \multirow{3}{*}{$\begin{array}{l}\mathrm{p}_{\mathrm{I}-\mathrm{II}}=0.0152 \\
\mathrm{p}_{\mathrm{I}-\mathrm{III}}=0.0176\end{array}$} & \multirow{3}{*}{$\begin{array}{l}\mathrm{p}_{\mathrm{I}-\mathrm{II}}=0.0043 \\
\mathrm{p}_{\mathrm{I}-\mathrm{III}}=0.0097\end{array}$} \\
\hline Nonalcoholic steatosis $(\mathrm{n}=20)$ & 73.68 & & \\
\hline NASH $(n=15)$ & 54.54 & & \\
\hline \multicolumn{4}{|c|}{ Stroke } \\
\hline No NAFLD (n=62) & 96.78 & \multirow{3}{*}{$\begin{array}{l}\mathrm{p}_{\mathrm{I}-\mathrm{II}}=0.0163 \\
\mathrm{p}_{\mathrm{I}-\mathrm{III}}=0.0058\end{array}$} & \multirow{3}{*}{$\begin{array}{l}\mathrm{p}_{\mathrm{I}-\mathrm{II}}=0.0057 \\
\mathrm{p}_{\mathrm{I}-\mathrm{III}}=0.0025\end{array}$} \\
\hline Nonalcoholic steatosis $(\mathrm{n}=20)$ & 74.14 & & \\
\hline NASH $(n=15)$ & 58.63 & & \\
\hline \multicolumn{4}{|c|}{ A repeated revascularization } \\
\hline No NAFLD (n=62) & 98.38 & \multirow{3}{*}{$\begin{array}{l}\mathrm{p}_{\mathrm{I}-\mathrm{II}}=0.0423 \\
\mathrm{p}_{\mathrm{I}-\mathrm{III}}=0.0286\end{array}$} & \multirow{3}{*}{$\begin{array}{l}\mathrm{p}_{\mathrm{I}-\mathrm{II}}=0.0146 \\
\mathrm{p}_{\text {I-III }}=0.0107\end{array}$} \\
\hline Nonalcoholic steatosis $(\mathrm{n}=20)$ & 82.80 & & \\
\hline NASH $(n=15)$ & 71.81 & & \\
\hline
\end{tabular}




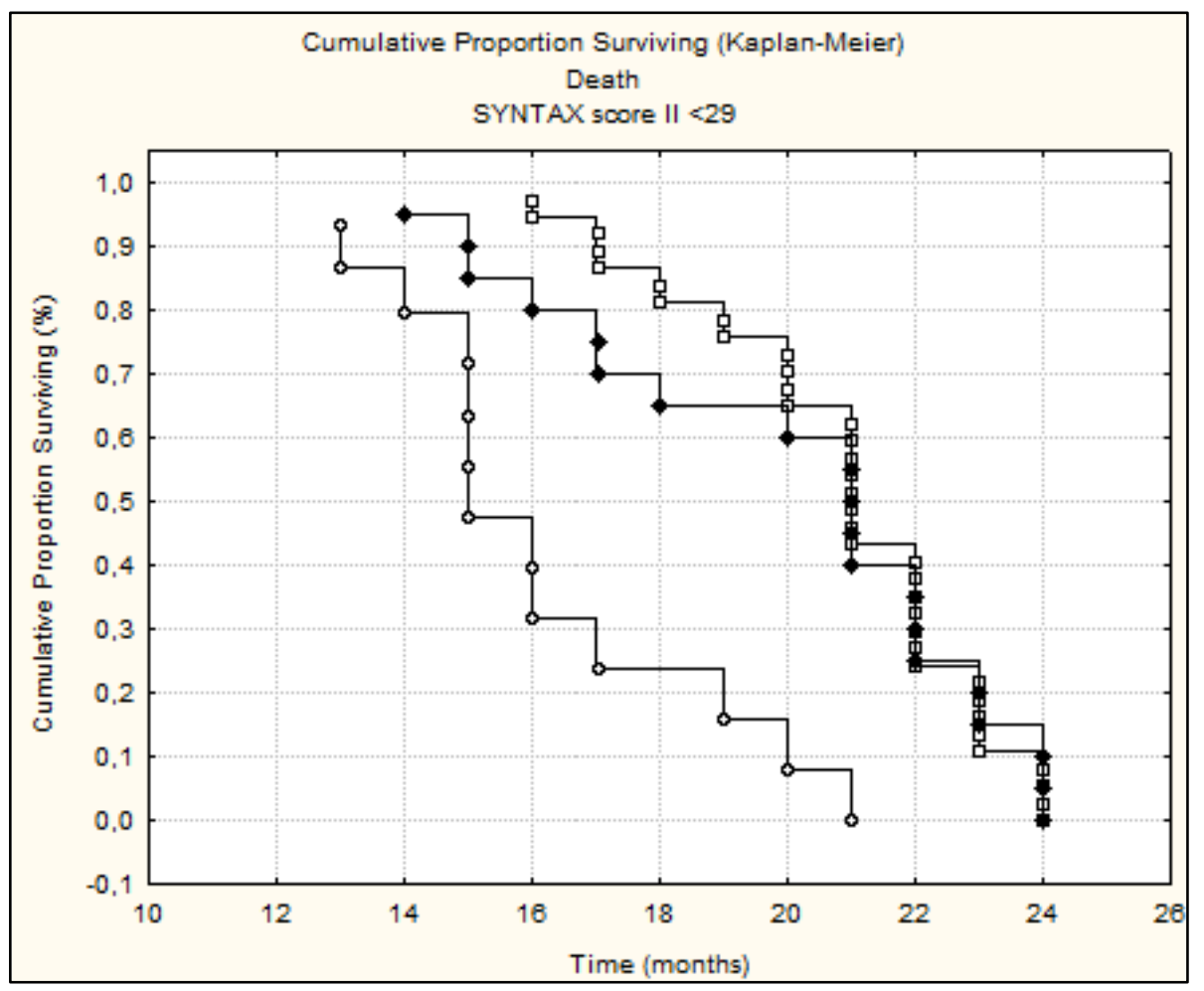

Figure 1. Kaplan-Meier survival curve for a death in patients with stable CHD with and without NAFLD at two-year follow-up (SS II <29)

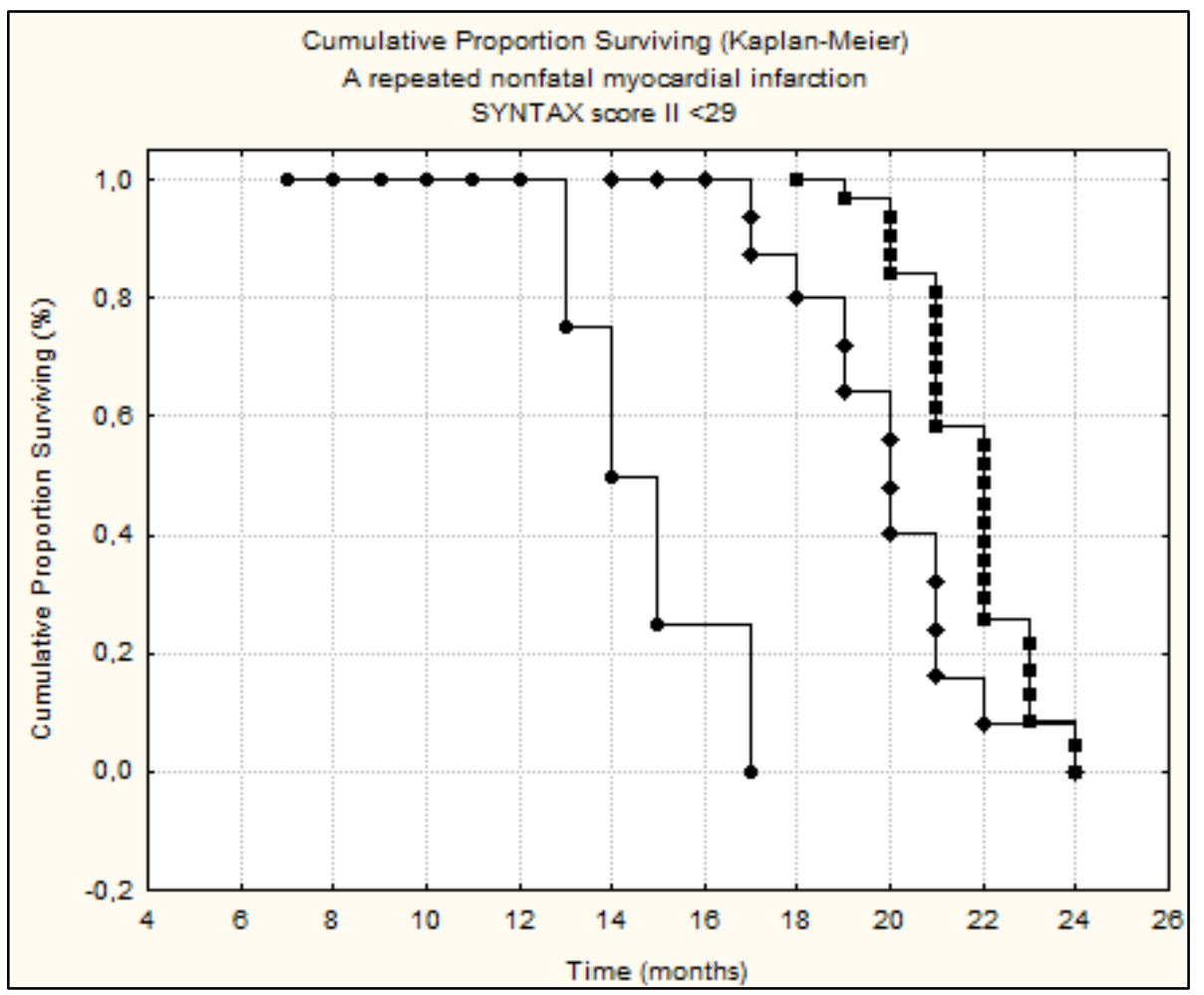

Figure 2. Kaplan-Meier survival curve for a repeated nonfatal myocardial infarction in patients with stable CHD with and without NAFLD at two-year follow-up (SS II <29) 


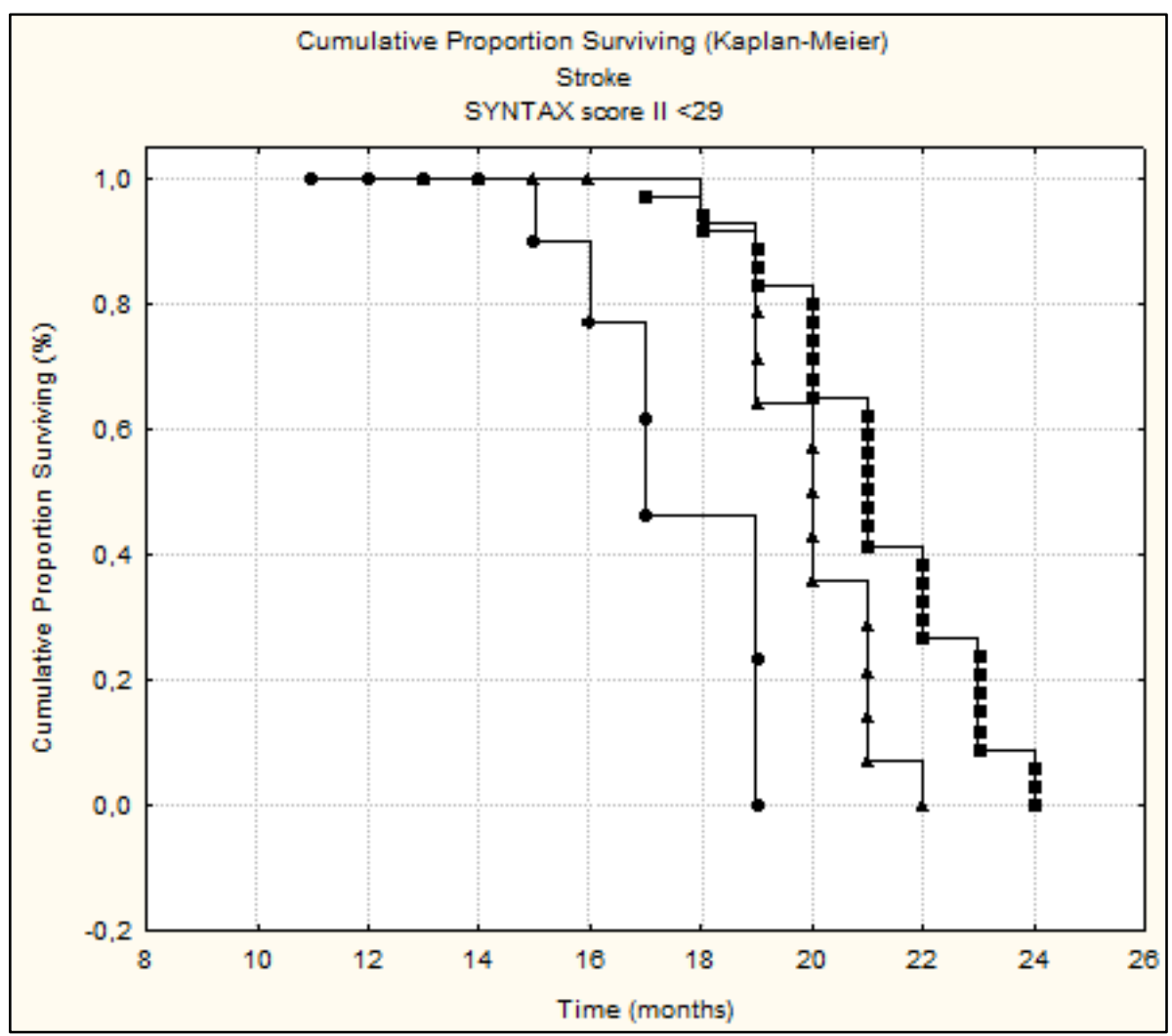

Figure 3. Kaplan-Meier survival curve for a stroke survival in patients with stable CHD with and without NAFLD at two-year follow-up (SS II $<29$ )

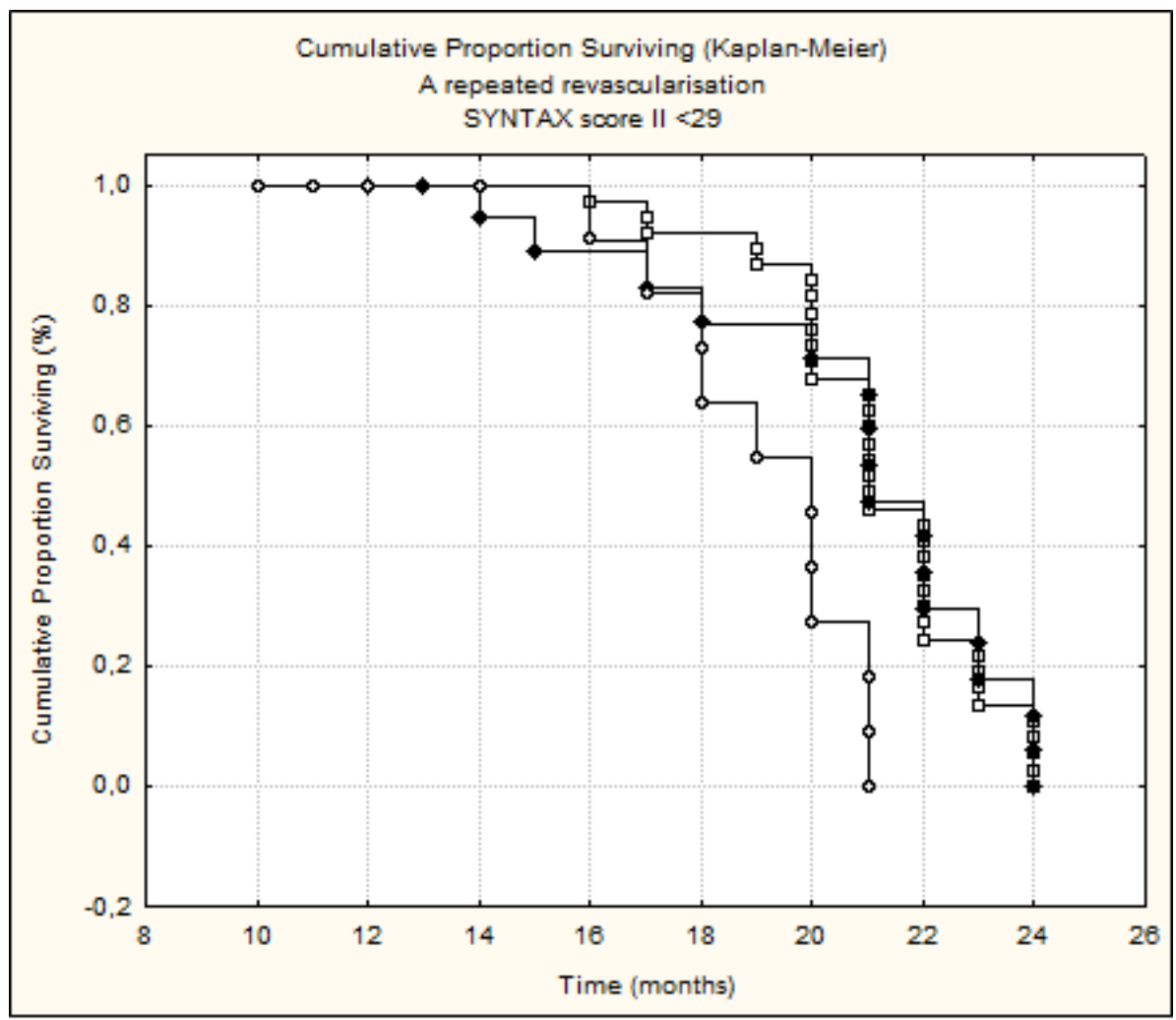

Figure 4. Kaplan-Meier survival curve for a repeated revascularization in patients with stable CHD with and without NAFLD at two-year follow-up (SS II <29) 
Table 3. A two-year survival analysis according to the NAFLD severity in patients with stable CHD with SYNTAX score II $\geq 29$

\begin{tabular}{|c|c|c|c|}
\hline \multirow{2}{*}{$\begin{array}{c}\text { A two-year } \\
\text { follow-up outcome }\end{array}$} & \multirow{2}{*}{ Cumulative proportion, $\%$} & \multicolumn{2}{|c|}{$P$ value } \\
\hline & & Gehan's Wilcoxon test & Cox's F test \\
\hline \multicolumn{4}{|c|}{ Death } \\
\hline No NAFLD $(n=26)$ & 100.00 & \multirow{3}{*}{$\begin{array}{l}\mathrm{p}_{\mathrm{I}-\mathrm{II}}=0.4728 \\
\mathrm{p}_{\mathrm{I}-\mathrm{III}}=0.0165\end{array}$} & \multirow{3}{*}{$\begin{array}{l}\mathrm{p}_{\mathrm{I}-\mathrm{II}}=0.2916 \\
\mathrm{p}_{\mathrm{I}-\mathrm{III}}=0.0068\end{array}$} \\
\hline Nonalcoholic steatosis $(\mathrm{n}=16)$ & 96.15 & & \\
\hline NASH $(n=8)$ & 60.00 & & \\
\hline \multicolumn{4}{|c|}{ A repeated nonfatal myocardial infarction } \\
\hline No NAFLD $(n=26)$ & 85.36 & \multirow{3}{*}{$\begin{array}{l}\mathrm{p}_{\mathrm{I}-\mathrm{II}}=0.0394 \\
\mathrm{p}_{\mathrm{I}-\mathrm{III}}=0.0045\end{array}$} & \multirow{3}{*}{$\begin{array}{l}\mathrm{p}_{\mathrm{I}-\mathrm{II}}=0.0203 \\
\mathrm{p}_{\mathrm{I}-\mathrm{III}}=0.0036\end{array}$} \\
\hline Nonalcoholic steatosis $(\mathrm{n}=16)$ & 49.54 & & \\
\hline NASH $(n=8)$ & 33.33 & & \\
\hline \multicolumn{4}{|c|}{ Stroke } \\
\hline No NAFLD $(n=26)$ & 88.95 & \multirow{3}{*}{$\begin{array}{l}\mathrm{p}_{\mathrm{I}-\mathrm{II}}=0.0137 \\
\mathrm{p}_{\mathrm{I}-\mathrm{III}}=0.0021\end{array}$} & \multirow{3}{*}{$\begin{array}{l}\mathrm{p}_{\mathrm{I}-\mathrm{II}}=0.0063 \\
\mathrm{p}_{\mathrm{I}-\mathrm{III}}=0.0017\end{array}$} \\
\hline Nonalcoholic steatosis $(\mathrm{n}=16)$ & 53.39 & & \\
\hline NASH $(n=8)$ & 37.85 & & \\
\hline \multicolumn{4}{|c|}{ A repeated revascularization } \\
\hline No NAFLD $(n=26)$ & 85.83 & \multirow{3}{*}{$\begin{array}{l}\mathrm{p}_{\mathrm{I}-\mathrm{II}}=0.0019 \\
\mathrm{p}_{\mathrm{I}-\mathrm{III}}=0.0006\end{array}$} & \multirow{3}{*}{$\begin{array}{l}\mathrm{p}_{\mathrm{I}-\mathrm{II}}=0.0013 \\
\mathrm{p}_{\mathrm{I}-\mathrm{III}}=0.0002\end{array}$} \\
\hline Nonalcoholic steatosis $(\mathrm{n}=16)$ & 58.80 & & \\
\hline NASH $(n=8)$ & 31.81 & & \\
\hline
\end{tabular}

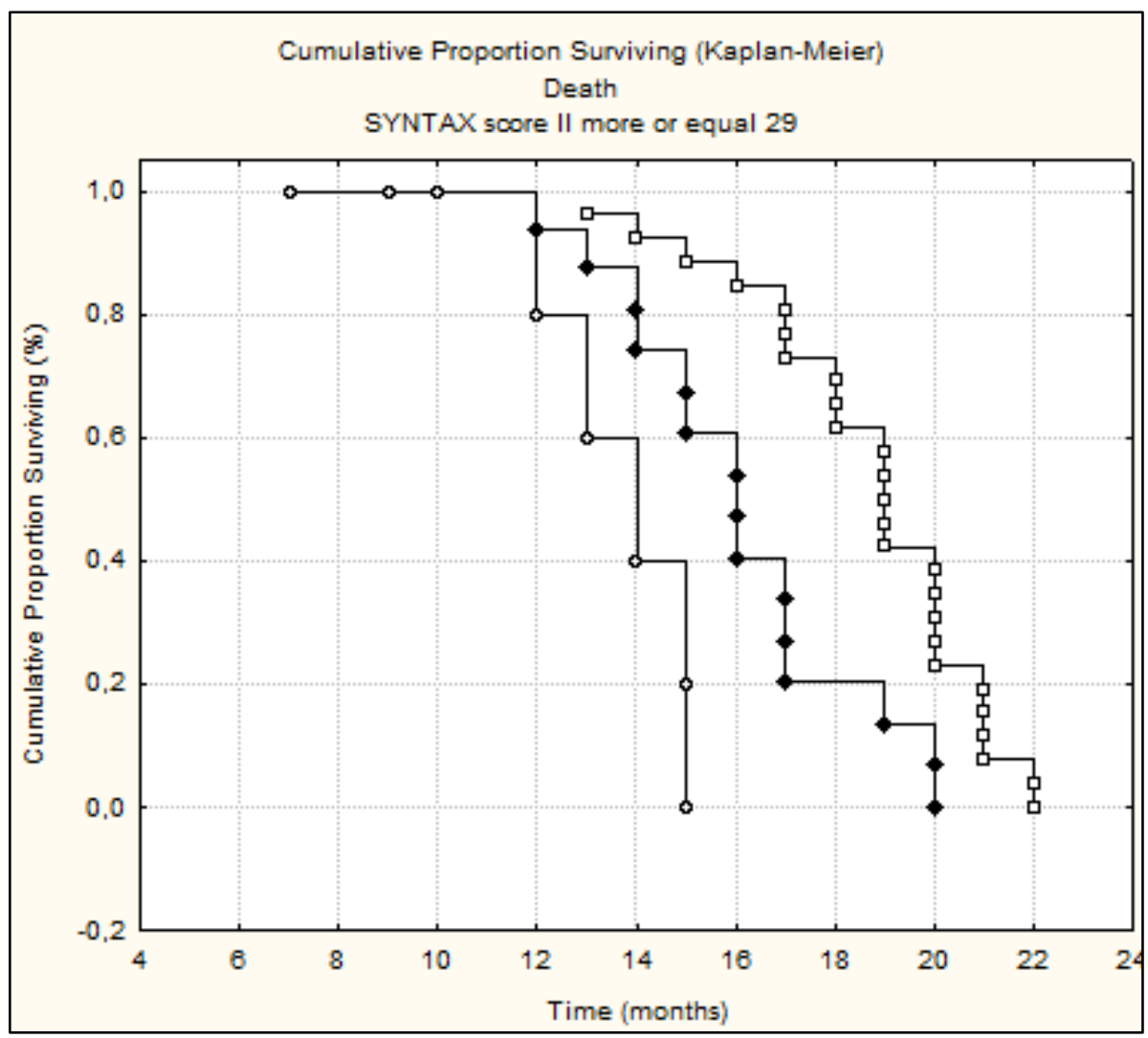

Figure 5. Kaplan-Meier survival curve for a death in patients with stable CHD with and without NAFLD at two-year follow-up (SS II $\geq 29$ ) 


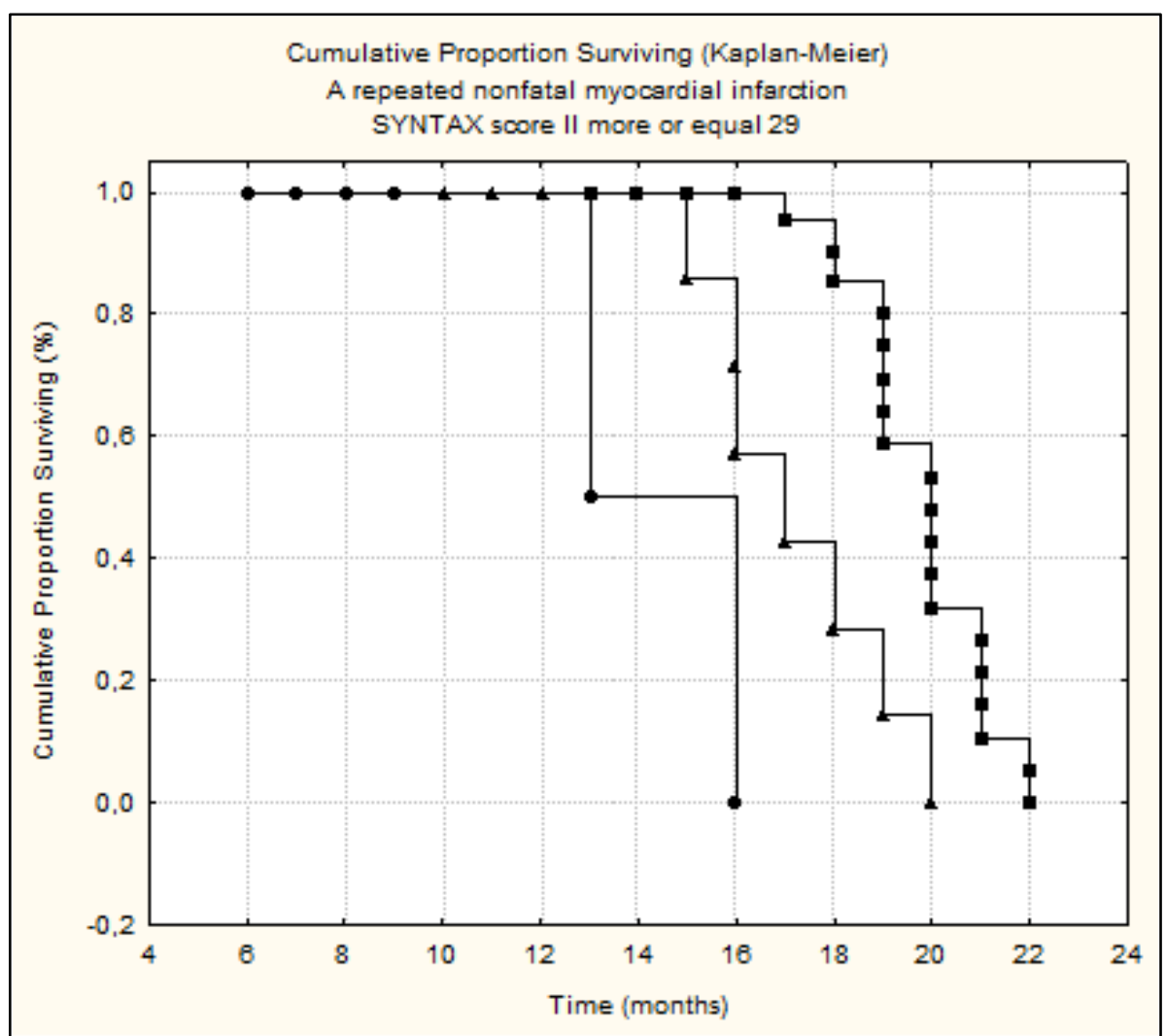

Figure 6. Kaplan-Meier survival curve for a repeated nonfatal myocardial infarction in patients with stable CHD with and without NAFLD at two-year follow-up (SS II $\geq 29$ )

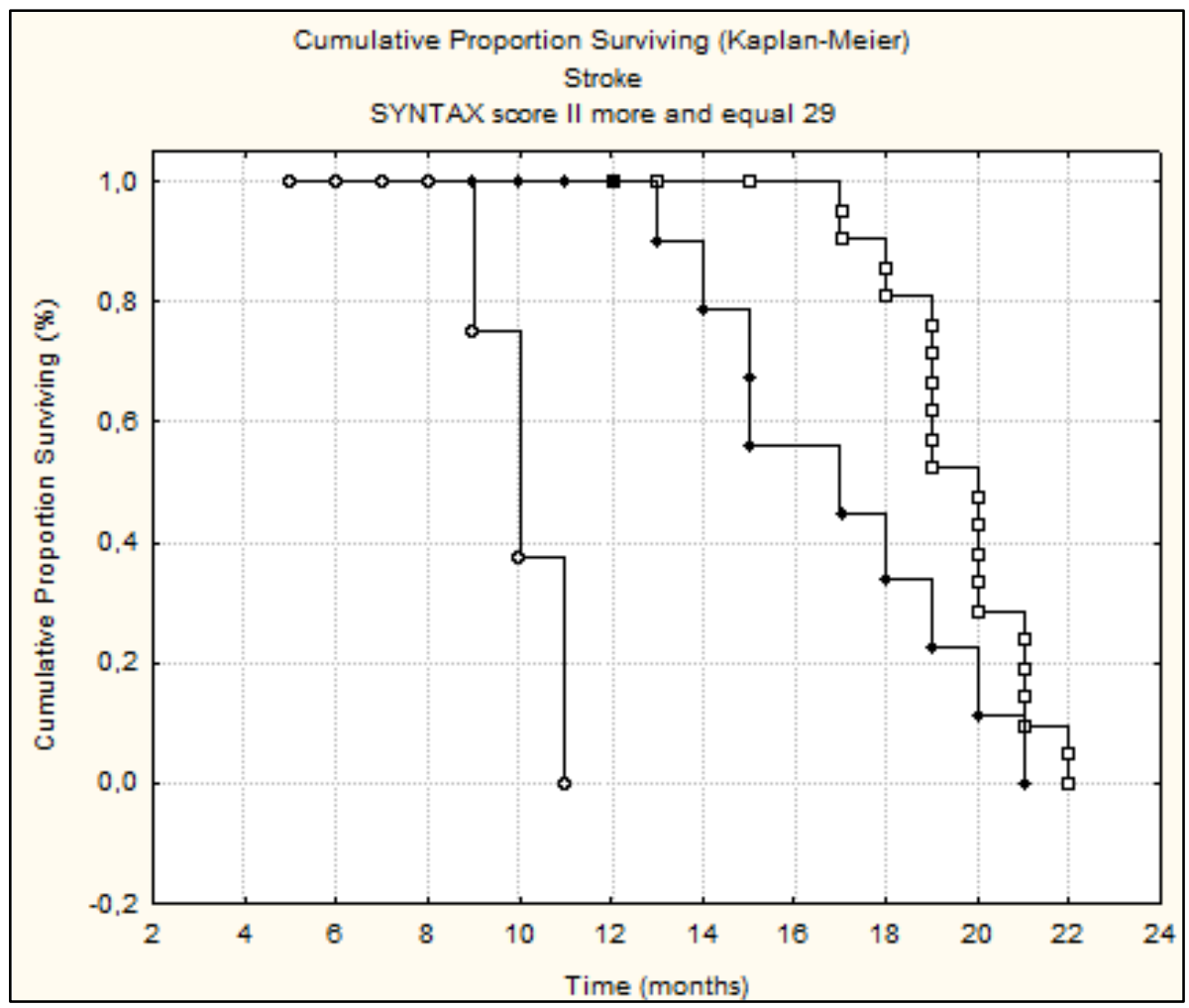

Figure 7. Kaplan-Meier survival curve for a stroke survival in patients with stable CHD with and without NAFLD at two-year follow-up (SS II $\geq 29$ ) 


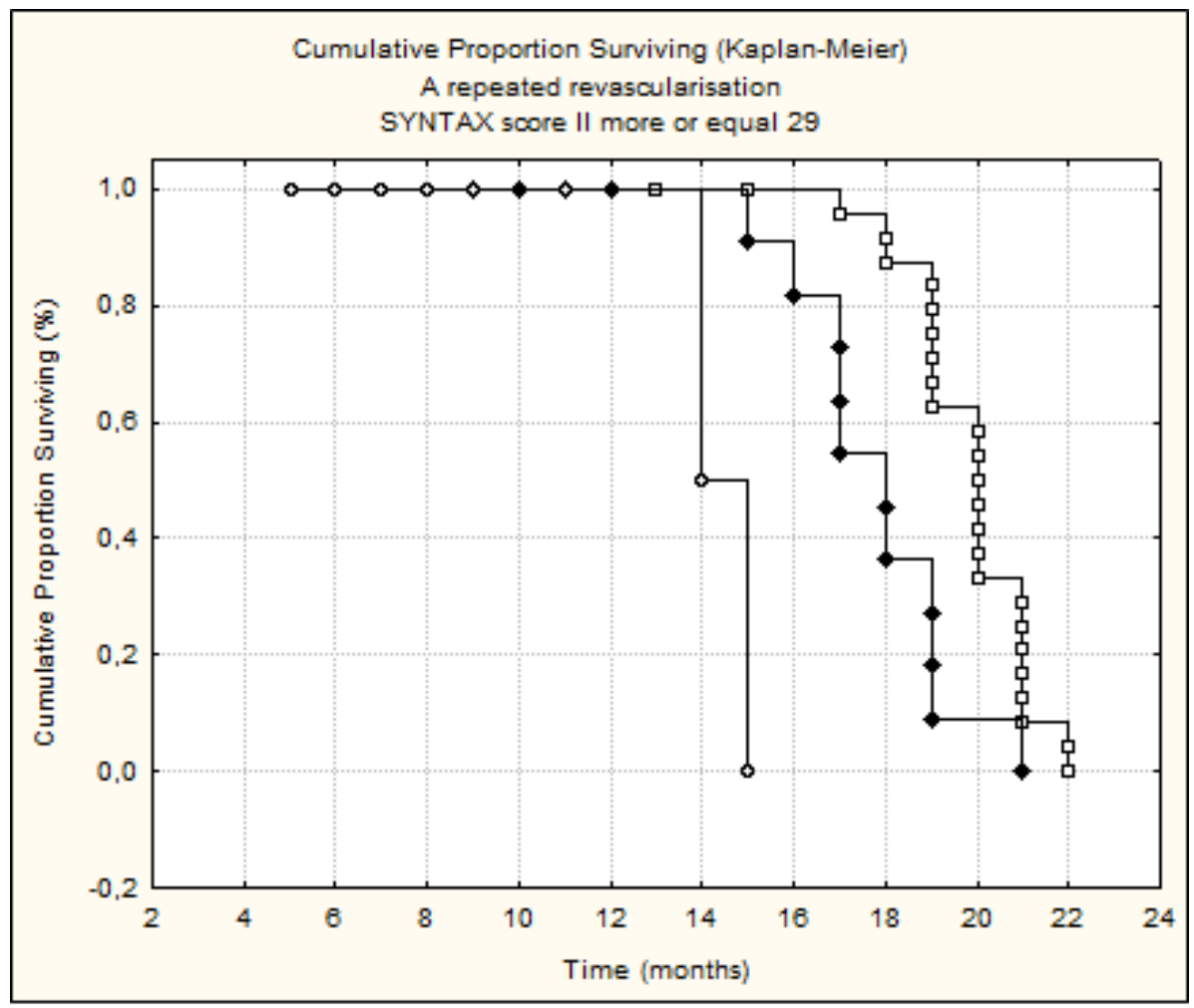

Figure 8. Kaplan-Meier survival curve for a repeated revascularization in patients with stable CHD with and without NAFLD at two-year follow-up (SS II $\geq 29)$

\section{Discussion}

Our study analyzed usefulness of SS II in the prediction of major cardiovascular events during two-year follow-up period in patients with stable CHD combined with NAFLD.

Currently use of SS II is a widespread all over the world due to its significance in choosing a treatment method of the patients with CHD and improving prognosis of these patients by consideration not only anatomical, but clinical features. This study revealed that the course of stable CHD in the long term perspective depends not only on the state of the coronary blood supply, heart contractility, creatinine clearance, presence of COPD, but the presence and the course of NAFLD.

Our results agree with the findings of the study carried out by Xu et al. [18]. That study found that the SS II for percutaneous coronary intervention was able to risk-stratify these patients and predict long-term adverse ischemic events, including death. Moreover, SS II demonstrated better predictability for long-term mortality compared with the strictly anatomic SS among this patients' subset.

These results are similar to the study performed by Azzarelli et al. where prognosis of the patients who underwent percutaneous coronary intervention in a high anatomical and clinical risk was associated with a high rate of major cardiovascular events [11]. These scientists revealed that the one-year death-free survival $(92.5 \%$ vs.
$100 \% ; \mathrm{p}=0.052)$ and myocardial infarction-free survival $(92.5 \%$ vs. $100 \% ; p=0.052)$ were lower in patients with high SS II than in those with low SS II.

According to Obeid et al. SS II provided predictive value for risk stratification, showing that the patients with high SS II had a 13-fold high risk for major cardiovascular events [19].

So, SS II represent a useful tool to predict the risk of adverse clinical events and to evaluate the interactions of risk factors, that could help in the decision making process between revascularization strategies. However, all these studies did not consider a liver state. Moreover, they did not anticipate the evaluation of fibrotic and immune-inflammatory changes in the liver structure.

Since, NAFLD was no longer consider as an isolated pathology of the hepatobiliary system, but an early predictor of cardiovascular disease and played a significant role in the evaluation of cardiovascular risk, our research included not only determination of the prognosis by SS II value, but stratification of the cardio-vascular risk taking into account the presence and severity of NAFLD.

The originality of our study was that we revealed that NAFLD significantly affects the prediction of patients with stable CHD and, accordingly, examination of liver state, contributes to a more thorough assessment of the prognosis of these patients. This, in turn, determines the prospects for finding ways to improve the long-term prognosis of the patients with a combined pathology by affecting the liver, in particular. 


\section{Conclusions}

The long-term prognosis of the patients with stable CHD depends not only on the anatomical and clinical variables that form SS II, but NAFLD course and is a most prognostically unfavorable on the background of SS II $\geq 29$ and NASH combination. Assessment of the SSII value considering the presence and severity of NAFLD might represent a useful tool to predict the risk of major cardiovascular events in patients with stable CHD.

\section{REFERENCES}

[1] M. T. Ağaç, L. Korkmaz, G. Çavuşoğlu. Association between Nonalcoholic Fatty Liver Disease and Coronary Artery Disease Complexity in Patients with Acute Coronary Syndrome, Angiology, Vol. 64, No. 8, 2013. Online available:

http://journals.sagepub.com/doi/pdf/10.1177/00033197134 79155

[2] S. Ballestri, A. Lonardo, S. Bonapace, C. D. Byrne, P. Loria, G. Targher. Risk of cardiovascular, cardiac and arrhythmic complications in patients with non-alcoholic fatty liver disease, World Journal of Gastroenterology, Vol. 20, P. 1724-1745, 2014.

[3] H. Chi-Sheng, T. Ping-Huei, T. Chia-Hung, C. Chien-Chuan, L. Wei-Chih, L. Yi-Chia. Nonalcoholic Fatty Liver Disease Is Associated With QT Prolongation in the General Population, Journal of the American Heart Association, Vol. 4, P. e001820, 2015. Online available: http://jaha.ahajournals.org/content/4/7/e001820/tab-figuresdata\#sec-17

[4] V. A. Chernyshov. Comorbid diseases affecting the cardiovascular risk in postinfarction patients, Ukrainian Therapeutic Journal, Vol. 2, P. 11-20, 2014.

[5] N. Alkhouri, A. J. McCullough. Noninvasive Diagnosis of NASH and Liver Fibrosis Within the Spectrum of NAFLD, Gastroenterology Hepatology, Vol. 8, No. 10, P. 661-668, 2012.

[6] A. Fouad, D. Sabry, R. Ahmed, M. Kamal, S. A. Allah, S. Marzouk. Comparative diagnostic study of biomarkers using FibroMax and pathology for prediction of liver steatosis in patients with chronic hepatitis $\mathrm{C}$ virus infection: an Egyptian study, International Journal of General Medicine, Vol. 6, P. 127-134, 2013.

[7] W. Wijns, P. Kolh, N. Danchin, C. Di Mario. The Task Force on Myocardial Revascularization of the European Society of Cardiology (ESC) and the European Association for Cardio-Thoracic Surgery (EACTS), European Association for Percutaneous Cardiovascular Interventions (EAPCI) Guidelines on myocardial revascularization, European Heart Journal, Vol. 31, P. 2501-2555, 2010.

[8] G. N. Levine, E. R. Bates, J. C. Blankenship, S. R. Bailey, J. A. Bittl, B. Cerce. ACCF/AHA/SCAI Guideline for Percutaneous Coronary Intervention: a report of the American College of Cardiology Foundation/American Heart Association Task Force on practice guidelines and the Society for Cardiovascular Angiography and Interventions,
Journal of American Colleague of Cardiology, Vol. 58, P. 44-122, 2011.

[9] A. Caixeta, P. Généreux, T. Palmerini. Prognostic utility of the SYNTAX score in patients with single versus multivessel disease undergoing percutaneous coronary intervention (from the Acute Catheterization and Urgent Intervention Triage StrategY [ACUITY] trial, American Journal of Cardiology, Vol. 113, P. 203-210, 2014.

[10] V. Farooq, D. van Klaveren, E. W. Steyerberg, E. Meliga, Y. Vergouwe, A. Chieffo. Anatomical and clinical characteristics to guide decision making between coronary artery bypass surgery and percutaneous coronary intervention for individual patients: development and validation of SYNTAX score II, Lancet, Vol. 381, P. 639650, 2013.

[11] S. Azzarelli, B. Marouane, S. Giubilato, S. D. Tomasello, M. Castaing, R. Giunta. Usefulness of SYNTAX score II in complex percutaneous coronary interventions in the setting of acute coronary syndrome, Journal of Saudi Heart Association, Vol. 28, No. 2, P. 63-72, 2016.

[12] S. L. Chen, Y. L. Han, Y. J. Zhang, F. Ye, H. W. Liu, J. J. Zhang. The anatomic- and clinical-based NERS (new risk stratification) score II to predict clinical outcomes after stenting unprotected left main coronary artery disease: results from a multicenter, prospective, registry study, JACC Cardiovascular Intervention, Vol. 6, P. 1233-1241, 2013.

[13] C. M. Campos, D. van Klavere, J. Iqbal, Y. Onuma, Y. J. Zhang, H. M. Garcia-Garcia. Predictive performance of SYNTAX Score II in patients with left main and multivessel coronary artery disease, Circulation Journal, Vol. 78, P. 1942-1949, 2014.

[14] V. V. Kravchenko, M. Yu. Sokolov, T. V. Talayeva. Unified Clinical Protocol "Stable coronary heart disease." $\mathrm{MOH}$ Ukraine Order \#152 from 03.02.2016. Online available: http://www.moz.gov.ua/docfiles/dn_20150716_1dod.pdf

[15] M. K. Xobzej, N. V. Xarchenko, O. M. Lishhyshyna. Unified Clinical Protocol "Non-alcoholic steatohepatitis". MOH Ukraine Order \#826 from 06.11.2014. Online available:

http://moz.gov.ua/docfiles/dn_20141106_0826_dod_ukp_n sg.pdf

[16] N. V. Kharchenko, O. M. Lishchyshyna, H. A. Anokhina. Adapted clinical guidelines based on evidence "Nonalcoholic fatty liver disease". Online available: http://www.moz.gov.ua/docfiles/dod_akn_dn_20140616_2. pdf

[17] Version 2.11 of the SYNTAX Score I and SYNTAX Score II calculator. Online available: http://www.syntaxscore.com/calculator/start.htm

[18] B. Xu, P. Genereux, Y. Yang, M. B. Leon, L. Xu, S. Qiao. Validation and comparison of the long-term prognostic capability of the SYNTAX score-II among 1,528 consecutive patients who underwent left main percutaneous coronary intervention, JACC Cardiovascular Intervention, Vol. 7, P. 1128-1137, 2014.

[19] S. N. Obeid, A. H. Frangieh, M. Jaguszewski, F. Mach, N. Rodondi, P. Vogt. Prognostic significance of syntax score II in patients presenting with acute coronary syndrome undergoing percutaneous intervention, Journal of American Colleague of Cardiology, Vol. 65, P. 45, 2015. 